MRS Advances (C) 2015 Materials Research Society

DOI: $10.1557 /$ adv.2015.6

\title{
GaSb and InSb Quantum Nanostructures: Morphologies and Optical Properties
}

\author{
Thanavorn Poempool ${ }^{1}, Z{ }^{1}$, Suwit Kiravittaya ${ }^{2}$, Suwat Sopitpan ${ }^{3}$, Supachok Thainoi ${ }^{1}$, Songphol Kanjanachuchai ${ }^{1}$, \\ Somchai Ratanathamaphan ${ }^{1}$, and Somsak Panyakeow ${ }^{1 *}$ \\ ${ }^{I}$ Semiconductor Devices Research Laboratory, Department of Electrical Engineering, Faculty of Engineering, \\ Chulalongkorn University, Bangkok 10330, Thailand \\ ${ }^{2}$ Department of Electrical and Computer Engineering, Faculty of Engineering, Naresuan University, \\ Phitsanulok 65000, Thailand \\ ${ }^{3}$ Thailand Microelectronics Center (TMEC), National Electronics and Computer Center, Chachoengsao 24000, \\ Thailand \\ ${ }^{*}$ Corresponding author. Tel.: +662-218-6524; Fax: +662-251-8991, \\ E-mail address: s_panyakeow@yahoo.com
}

\begin{abstract}
$\mathrm{GaSb} / \mathrm{GaAs}$ and $\mathrm{InSb} / \mathrm{GaAs}$ material systems can create type-II quantum nanostructures which provide interesting electronic and optical properties such as having long carrier life time, low carriers-recombination rate, and emitting/absorbing low photon energy. These characteristics of type-II nanostructures can be applied for infrared or gas detection devices, for memory devices and even for novel intermediate band solar cells. In contrast, lattice mismatches of $\mathrm{GaSb} / \mathrm{GaAs}$ and $\mathrm{InSb} / \mathrm{GaAs}$ material system are $7.8 \%$ and $14.6 \%$, respectively, which need some specific molecular beam epitaxial (MBE) growth conditions for quantum nanostructure formation via Stranski-Krastanov growth mode.

In this paper, the growth of self-assembled $\mathrm{GaSb}$ and InSb quantum nanostructures on (001) GaAs substrate by using MBE was reported. The surface morphology of these two quantum nanostructures and their optical properties were characterized by atomic force microscopy and photoluminescence (PL). The experimental results were compared between these two quantum nanostructures. Due to the lattice mismatch in each material system and the difference in sticking coefficient of Ga- and In-atoms during epitaxial growth, we obtain $\mathrm{GaSb} / \mathrm{GaAs}$ quantum dots (QDs) with a density $\sim 10^{10} \mathrm{dots} / \mathrm{cm}^{2}$ and $\mathrm{InSb} / \mathrm{GaAs}$ QDs with a density of $\sim 10^{8} \mathrm{dots} / \mathrm{cm}^{2}$. The facet analysis of individual quantum nanostructure in each material system reveals that $\mathrm{GaSb} / \mathrm{GaAs} \mathrm{QD}$ has a dome-like shape with nearly isotropic property while InSb QDs form a rectangular-like shape with elongation along [110]-direction showing a strong anisotropic property.
\end{abstract}

Low temperature PL spectra from capped GaSb and InSb quantum nanostructures show the energy peaks at 1.08-1.11 and 1.16-1.17 eV, respectively. The variations of PL peaks as a function of both temperature and excitation power are investigated. PL peak shows clear blue shift when excitation power is increased. This work manifests a possibility to use both GaSb and InSb quantum nanostructures for nanoelectronic and nanophotonic applications. 


\section{INTRODUCTION}

$\mathrm{GaSb} / \mathrm{GaAs}$ quantum nanostructure can be realized by molecular beam epitaxy (MBE) via Stranski-Krastanov growth mode. The lattice mismatching of $\mathrm{GaSb} / \mathrm{GaAs}$ material system is $7.8 \%$ which is similar to that of $\mathrm{InAs} / \mathrm{GaAs}$. However, $\mathrm{GaSb} / \mathrm{GaAs}$ is type II nanostructure where only holes are quantized but electron are loosely bound to the interface of $\mathrm{GaSb}$ and GaAs[1]. The carrier separated confinement in this type II nanostructure provides long carrier life time which is beneficial for memory application [2].

On the other hand, InSb/GaAs material system has larger lattice mismatch of $14.6 \%$. Under specific MBE growth parameters, $\mathrm{InSb} / \mathrm{GaAs}$ nanostructure is also able to be grown. $\mathrm{InSb} / \mathrm{GaAs}$ is in the same category of type II nanostructure but with much higher electron mobility. High speed behavior of this nanoelectronic device is another key merit. InSb/GaAs quantum nanostructure is still not well known. Very few researches on $\mathrm{InSb} / \mathrm{GaAs}$ are conducted [3-4].

In this work, we investigate the MBE growth parameters of $\mathrm{GaSb} / \mathrm{GaAs}$ and $\mathrm{InSb} / \mathrm{GaAs}$ nanostructures. We found that the surface morphologies of these two material systems are quite different. GaSb/GaAs system provides quantum dot (QD) structure with dome shape and circular base similar to that of InAs/GaAs QD. However, InSb/GaAs system creates an interesting shape with rectangular base having an elongation along [110] crystallographic direction.

Photoluminescence (PL) measurements of these two quantum nanostructures are conducted at varying temperatures from $20 \mathrm{~K}$ up to $100 \mathrm{~K}$. Excitation power dependence of PL intensities confirms that both quantum nanostructures are type-II. Potential applications of these two quantum nanostructures are discussed.

\section{MBE GROWTH OF GaSb/GaAs AND InSb/GaAs QUANTUM NANOSTRUCTURES}

Both $\mathrm{GaSb}$ and InSb quantum structures were carried out by solid-source MBE using Riber Compact 21T system. Semi-insulating (001) GaAs substrate was used for all growth runs. After oxides had removed out of the substrate surface by thermal preheating, the As 4 source cell was heated up to be diffused the entire growth chamber. Next, the substrate surface was flattened out $500 \mathrm{~nm} \mathrm{GaAs}$ buffer layer at $580^{\circ} \mathrm{C}$. At this stage onward, reflection high-energy electron diffraction (RHEED) pattern was observed to study surface transformation during the growth. Then, the substrate temperature was decreased to $450^{\circ} \mathrm{C}$ for GaSb QDs formation and much low temperature of $260^{\circ} \mathrm{C}$ for InSb QDs formation. After that, temperature of As-cell was reduced to keep the chamber out of $\mathrm{As}_{4}$ atoms. Performing antimony dots growth would be done when background pressure reached down below 5.0 $\times 10^{-9}$ torr. To start GaSb growth, the entire chamber was soaking by $\mathrm{Sb}_{4}$ for 1 minute and then $\mathrm{Ga}$-shutter was open. $\mathrm{Ga}$-atoms with the 
growth rate of 0.14 monolayers per second (ML/s) interacted with Sb-atoms on the GaAs surface resulted in 6.0 ML QDs construction which confirmed by RHEED pattern transformation. Gaand $\mathrm{Sb}_{4}$ cracker- shutters were closed simultaneously when RHEED pattern became spotty. For 3.0 ML InSb QDs, in contrast, In-shutter with the growth rate of $0.01 \mathrm{ML} / \mathrm{s}$ was left opening for 5 minute after 1 minute antimony soaking and simultaneously close when the time was due. $\mathrm{GaSb}$ and InSb quantum structures were buried under GaAs layer of $200 \mathrm{~nm}$ at $450^{\circ} \mathrm{C}$ and $260^{\circ} \mathrm{C}$ for $\mathrm{GaSb}$ and $\mathrm{InSb}$, respectively, for PL study.

Optical characterization and morphology were performed by PL measurement and atomic force microscopy (AFM). PL was measured in a vacuum cryostat and the temperature was varied from $20 \mathrm{~K}$ to over $100 \mathrm{~K}$. The samples of $\mathrm{GaSb}$ and $\mathrm{InSb}$ quantum structure were excited by $514.5 \mathrm{~nm} \mathrm{Ar}^{+}$laser with a power variation from $16 \mathrm{~mW}$ to $96 \mathrm{~mW}$. PL signal of power- and temperature-dependent were detected by an $\mathrm{LN}_{2}$-cooledInGaAs detector with a lock-in technique.

\section{EXPERIMANTAL RESULTS}
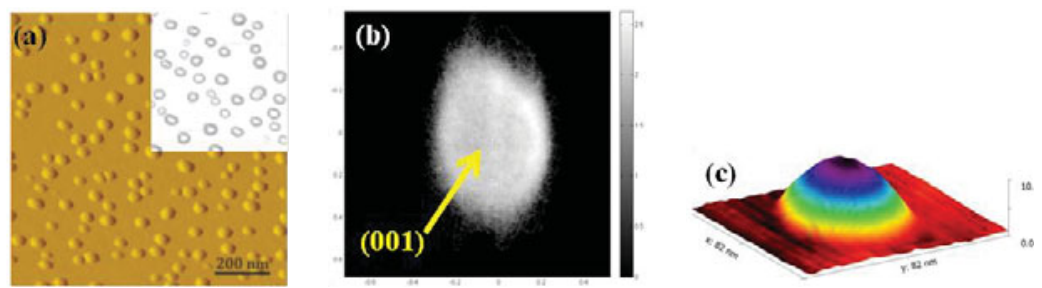

Fig. 1. (a) $1 \times 1 \mu \mathrm{m}^{2}$ AFM image of GaSb QDs/GaAs, (b) a facet plot of the corresponding AFM image shown in (a) and (c) its dome like shape GaSb QDs.
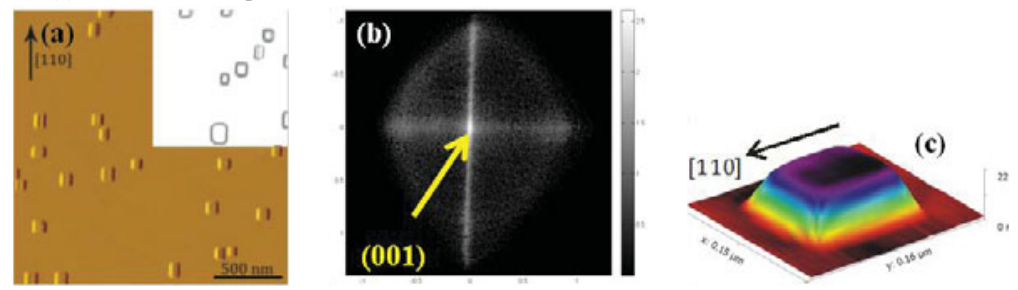

Fig. 2. (a) $2 \times 2 \mu \mathrm{m}^{2} \mathrm{AFM}$ image of GaSb QDs/GaAs, (b) facet plot of the corresponding AFM image shown in (a), (c) single InSb QD with rectangular based-shape..

The morphology GaSb and InSb quantum structures were characterized by AFM. Figures 1 (a) and 2 (a) show the $1 \times 1 \mu \mathrm{m}^{2}$ and $2 \times 2 \mu \mathrm{m}^{2}$ AFM images of GaSb QDs and InSb QDs grown on GaAs surface, respectively. According to the images, it is obviously seen that 6.0 ML GaSb QDs have a high areal density $\sim 10^{10} \mathrm{dots} / \mathrm{cm}^{2}$. In contrast, the areal density of 3.0 ML InSb QDs is 
less than of $\mathrm{GaSb}$ dots around 2 orders of magnitude, which can be counted $\sim 10^{8}$ dots $/ \mathrm{cm}^{2}$. This can be taken into account that the different misfit percentage in each material system can give rise in the different in areal density. As we can see in the facet analysis [5], the shape of $\mathrm{GaSb}$ (Fig. 1 (b)) is a circular base with a dome like-shape in 3D image of Fig. 1 (c), while the facet analysis of InSb QD and its single dot shape, shown in Fig. 2 (b) and 2 (c), has rectangular based-shape aligned parallel to [110]- direction.

The statistical information of $\mathrm{GaSb}$ and $\mathrm{InSb}$ quantum structures revealed their heights are $6.5 \pm$ $1.1 \mathrm{~nm}$ and $20.1 \pm 1.5 \mathrm{~nm}$, respectively, diameters of $\mathrm{GaSb}$ is $87.1 \pm 8.9 \mathrm{~nm}$ and length of $\mathrm{InSb}$ in [110] and [1-10] are $109.0 \pm 17.0 \mathrm{~nm}$ and $98.9 \pm 14.1 \mathrm{~nm}$, respectively.
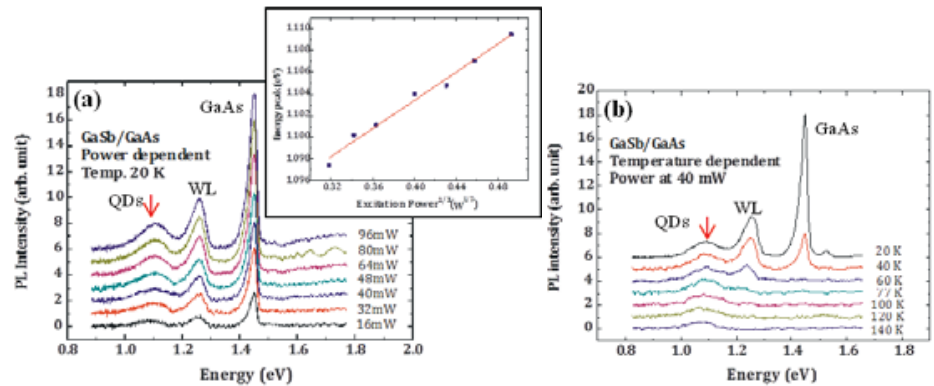

Fig. 3. (a) Power dependent PL with the energy peak $\sim 1.10-1.11 \mathrm{eV}$. The inset indicates the linear plot between $P^{1 / 3}$ and $E_{\text {peak }}$ and (b) temperature dependent PL spectra.
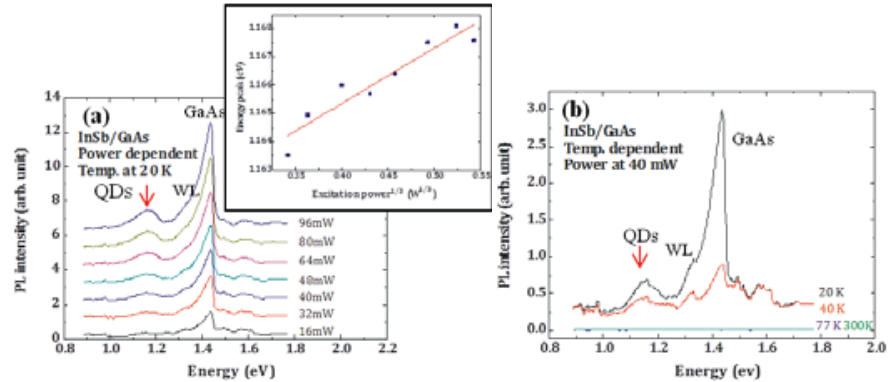

Fig. 4. (a) Power dependent PL with the energy peak $\sim 1.16 \mathrm{eV}$. The inset indicates the linear plot between $P^{1 / 3}$ and $E_{\text {peak }}$ and (b) temperature dependent PL spectra.

Optical properties of GaSb and InSb QDs are shown in Fig. 3 and 4. The PL spectra of both structures mainly composed of three peaks, as they are QDs peak, wetting layer peak and GaAs peak. According to the figures, it seems that InSb wetting layer peak and GaAs peak of InSb are merged together. This is caused by a very large in lattice mismatch between InSb and GaAs. 
Figures 3 (a) and 4 (a) show the power-dependent PL spectra at $20 \mathrm{~K}$ of GaSb QDs and InSb QDs which their energy peaks at $40 \mathrm{~mW}$ are $1.10 \mathrm{eV}$ and $1.16 \mathrm{eV}$, respectively. The increasing of excitation power $P$ gives rise in intensity since the excited carriers are being increased. Blueshift of PL peak energy $E_{\text {peak }}$ is observed. The plot between $P^{1 / 3}$ against $E_{\text {peak }}$ of both structures, shown in the insets of Fig. 3 (a) and 4 (a), confirmed the type-II characteristic by having a linear relation [6]. In addition, the plot of temperature-dependent of both structures measured at $40 \mathrm{~mW}$ shown in Fig. 3 (b) and 4 (b), respectively. For GaSb QDs, the intensity peaks dropped steadily reversed to the increased temperatures, whereas the intensity peaks of InSb QDs dropped more dramatically than of GaSb QDs. The blue-shift in temperature dependence PL can be explained by the increasing number of thermal-excited carriers leads to a triangular potential well where the electron quantization energy is lifted up [6].

The blue-shift of PL spectra indicated that GaSb QDs and InSb QDs are type-II quantum structure. The separation between electrons and holes in different materials causes the less carrier recombination rate which electrons can freely move toward the bulk and result in a long carrier lifetime. These fundamental properties of type-II is a great potential for high efficiency and fast operation devices such as low energy photon absorption solar cell, IR detector, laser and memory devices.

\section{CONCLUSIONS}

We have realized GaSb and InSb quantum structures on (001) GaAs substrate by MBE. We have studied their morphologies, facets and optical properties. The work shows a promising trend to apply type-II nanostructure in potential applications like IR-related devices.

\section{ACKNOWLEDGEMENTS}

This study was funded by the Research Chair Grant, the National Science and Technology Development Agency (NSTDA), Thailand, Thailand Research Fund (TRF), Nanotechnology Center of Thailand (NANOTECH), Asian Office of Aerospace Research and Development (AOARD) (Grant No. FA2386-14-1-4081), Ratchadapisek Sompoch Endowment Fund (2015), Chulalongkorn University (CU-58-051-EN) and AUN/SEED-NET.

\section{REFERENCES}

[1] H. Kroemer, Rev. Mod. Phys., 73 (3), (2001).

[2] M. Hayne, R.J. Young, E.P. Smakman, T. Nowozin, P. Hoidgson, J.K. Garleff, P. Rambabu, P.M. Koenraad, A. Marent, L. Bonato, A. Schliwa, D. Bimberg, J. Phys. D: Appl. Phys., 46, 264001 (2013).

[3] B.R. Bennett, P.M. Thibado, M.E. Twigg, E.R. Glaser, R. Magno, B.V. Shanabrook L.J. Whitman, J. Vac. Sci. Technol. B, 14(3), (1996). 
[4] A.F. Tsatsul'nikov, N.N. Ledentsov, M.V. Maksimov, B.Y. Mel'tser, P.V. Neklyudov, S.V. Shaposhnikov, B.V. Volovik, I.L. Krestnikov, N.A.Bert, P.S. Kop'ev, Zh.I. Alferov and D. Bimberg, Semiconductore 31 (1), 55-58 (1997).

[5] G. Costantini, A. Rastelli, C. Manzano, R. Songmuang, O.G. Schmidt, K. Kern and H. von Känel, Appl. Phys. Lett. 85, 5673 (2004).

[6] N.N. Ledentsov et.al., Phys. Rev. B 52, 14058 (1995). 